\title{
Integration of early supportive and palliative care in a patient's journey with cancer
}

DOI:

10.1080/09699260.2019.1659658

\section{Document Version}

Accepted author manuscript

Link to publication record in Manchester Research Explorer

\section{Citation for published version (APA):}

Lyon, J., Cook, N., \& Lawrie, I. (2019). Integration of early supportive and palliative care in a patient's journey with cancer. Progress in Palliative Care, 27(5), 206-212. https://doi.org/10.1080/09699260.2019.1659658

\section{Published in:}

Progress in Palliative Care

\section{Citing this paper}

Please note that where the full-text provided on Manchester Research Explorer is the Author Accepted Manuscript or Proof version this may differ from the final Published version. If citing, it is advised that you check and use the publisher's definitive version.

\section{General rights}

Copyright and moral rights for the publications made accessible in the Research Explorer are retained by the authors and/or other copyright owners and it is a condition of accessing publications that users recognise and abide by the legal requirements associated with these rights.

\section{Takedown policy}

If you believe that this document breaches copyright please refer to the University of Manchester's Takedown Procedures [http://man.ac.uk/04Y6Bo] or contact uml.scholarlycommunications@manchester.ac.uk providing relevant details, so we can investigate your claim.

\section{OPEN ACCESS}




\section{Progress in Palliative Care}

\section{Integration of early supportive and palliative care in a patient's journey with cancer --Manuscript Draft--}

Manuscript Number:

Full Title:

Article Type:

Keywords:

Corresponding Author:

\section{PPC363R1}

Integration of early supportive and palliative care in a patient's journey with cancer

Narrative Review

Palliative Care; Cancer; supportive care; early intervention

lain Lawrie

Pennine Acute Hospitals NHS Trust

UNITED KINGDOM

Corresponding Author Secondary Information:

Corresponding Author's Institution:

Pennine Acute Hospitals NHS Trust

Corresponding Author's Secondary Institution:

First Author:

Jack Lyon

First Author Secondary Information:

Order of Authors:

Jack Lyon

Natalie Cook

lain Lawrie

Order of Authors Secondary Information:

Abstract:

Specialist Palliative Care aims to effectively support the quality of life of patients and those close to them through progressive, life-limiting disease. Quality of life, an individual concept, requires a personalised approach to support and maintain it. Primarily achieved through the management of symptoms, both physical and psychological, alongside social and spiritual support, this approach is of the utmost importance to patients with advanced malignancy.

Several randomised, controlled trials suggest earlier provision of specialist palliative care may increase quality of life, improve symptoms and facilitate considered end of life care planning. This appears beneficial; however, evidence is mixed about the effectiveness of early specialist palliative care and its potential benefits. Results, therefore, should be interpreted with caution.

In reviewing the literature, it is clear that implementing early specialist palliative care is fraught with obstacles and requires increased resources and funding. Until the benefits and cost implications for such provision are better understood, it will not be accessible to all that may have potential to benefit.

Funding Information: 


\section{Integration of early supportive and palliative care in a patient's journey with cancer} Jack Lyon ${ }^{1}$, Natalie Cook$^{2}$, Iain Lawrie ${ }^{3}$

1. Undergraduate Medical Student, The University of Manchester, Faculty of Biology, Medicine and Health, Oxford Road, Manchester, United Kingdom M13 9PL

2. Senior Clinical Lecturer in Experimental Cancer Medicine and Honorary Consultant in Medical Oncology, University of Manchester, Division of Cancer Sciences, Faculty of Biology, Medicine and Health and The Christie NHS Foundation Trust, Wilmslow Road, Manchester, United Kingdom M20 4BX

3. Consultant and Honorary Clinical Senior Lecturer in Palliative Medicine, The Pennine Acute Hospitals NHS Trust / The University of Manchester, North Manchester General Hospital, Delaunays Road, Manchester, United Kingdom M8 5RB

Corresponding author:

Dr Iain Lawrie, North Manchester General Hospital, Delaunays Road, Manchester M8 5RB.

Telephone: $01617202972 \quad$ E-mail: iain.lawrie@pat.nhs.uk

Key words: $\quad$ palliative care, cancer, supportive care, early intervention

Conflicts of interest: IL is President of the Association for Palliative Medicine of Great Britain and Ireland

Word count: $\quad$ Abstract: 166; Body text: 3401

Author contributions: The authors confirm that all authors listed meet all four of the criteria recommended by the ICMJE. 
Integration of early supportive and palliative care in a patient's journey with cancer: a review

\begin{abstract}
Specialist Palliative Care aims to effectively support the quality of life of patients and those close to them through progressive, life-limiting disease. Quality of life, an individual concept, requires a personalised approach to support and maintain it. Primarily achieved through the management of symptoms, both physical and psychological, alongside social and spiritual support, this approach is of the utmost importance to patients with advanced malignancy.
\end{abstract}

Several randomised, controlled trials suggest earlier provision of specialist palliative care may increase quality of life, improve symptoms and facilitate considered end of life care planning. This appears beneficial; however, evidence is mixed about the effectiveness of early specialist palliative care and its potential benefits. Results, therefore, should be interpreted with caution.

In reviewing the literature, it is clear that implementing early specialist palliative care is fraught with obstacles and requires increased resources and funding. Until the benefits and cost implications for such provision are better understood, it will not be accessible to all that may have potential to benefit. 


\section{Introduction}

"The test of a civilisation is in the way that it cares for its helpless members".

$(\text { Buck, 1954) })^{1}$

One group of society's most "helpless members" includes those burdened with advanced disease and whose illness is not amenable to cure. This group is wide and disparate but includes patients diagnosed with malignant disease, whose illness journey may be short or can stretch to many years. No matter the course or timescale, the needs of cancer patients can be great and the quality of life (QoL) they experience can depend on the support they receive.

Popular opinion appears to be that palliative care (PC) is associated with death. Patients associate supportive care with psychological and social support; yet associate PC with endof-life care. $^{2}$ While partially true, palliation of symptoms is also appropriate throughout potentially curative treatment. This misperception may have a negative impact on the involvement of Specialist Palliative Care services (SPC) in patients' care. Physicians, nurses and other allied healthcare professionals also struggle with such differences in interpretation, and referrals to SPC can be hampered by this. ${ }^{3}$ This suggests a need for "re-branding" and a better "marketing" of SPC.

SPC is a distinct medical specialty, as opposed to generalist PC that is provided, often to a very high standard, by all doctors and healthcare professionals, but most notably by General Practitioners and other community-based professionals. ${ }^{4}$

To palliate originates from the Latin pallium, meaning "to cloak". SPC philosophy holds true 
to this; to cloak the symptoms and problems of those with life-limiting illness. The fact that SPC originated in cancer care and this is still its main focus is unsurprising, as one in six deaths globally is the result of cancer and, at least at the specialty's inception, treatments available for this group of patients were few. ${ }^{5}$

While SPC in the management of cancer has developed to provide expert input at the end of life, it has recently been recognised that SPC may be applicable much earlier: at diagnosis, through potentially curative treatment and at appropriate points throughout the course of malignant disease. ${ }^{6}$ Effective PC aims to achieve the best QoL possible and it seems obvious that this would be beneficial; so would earlier integration of SPC be beneficial to patients, and those close to them, in their journey with cancer? ${ }^{7}$

To explore this, we first investigate the meaning of Specialist Palliative Care and the other names given to this discipline. We then look into what drives progress in the field of SPC and what guidelines are available to healthcare professionals. Following this, we look at what difference, if any, can be made by incorporating palliative care earlier into a patient's journey by examining the research based around improving quality of life, symptom management, survival and end of life planning. We conclude this article by considering potential barriers to earlier integration and what conclusions can be drawn from our research.

\section{Specialist Palliative Care / Supportive and Palliative Care}

SPC and Supportive and Palliative Care are in essence the same and, indeed, the National Cancer Institute attributes the same definition to both. ${ }^{8}$ The existence of both terms could be understood in Figure 1, where it suggests how Supportive Care and Palliative Care could be applicable at different points in a patient's progressively deteriorating condition (Figure 1). 
SPC, according to the General Medical Council, is "the holistic care of persons with advanced, progressive, incurable illness", specifically aimed towards managing patients' symptoms and side effects of treatment. ${ }^{9}$ Management is based on the model of 'total pain', where not only physical symptoms but also psychological, social and spiritual issues are addressed $^{10}$ and support for those close to the patient is equally important. This may appear to be a 'non-medical' approach, but if the healthcare team doesn't acknowledge, explore and address such issues, medical actions and interventions to address physical issues may be rendered futile. Additionally, advance care planning should be initiated early in the patient journey and be a continuous process. ${ }^{11}$ It is clear, then, that in order to achieve 'total pain' management, a multidisciplinary team of doctors, nurses, allied health professionals and those who represent religious and other beliefs are required.

\section{Drivers}

How those approaching the end of their lives are cared for is an indicator of how we care for all sick and vulnerable people in our society. ${ }^{12}$ The last decade has seen an increasing awareness and interest in palliative and end of life care and recognition of the importance of 'generic' PC.

The Gold Standards Framework and Macmillan GP Facilitator programmes aimed to improve the PC of patients in the community and improve access to educational opportunities for primary care professionals. ${ }^{12,13}$ National initiatives, including A High Quality Workforce: NHS Next Stage Review and the End of Life Care Strategy identified the importance of further development of PC in the UK. ${ }^{14-16}$ Secondary care based initiatives, such as the 
Routes to Success in End of Life Care and the Transforming End of Life Care in Acute Hospitals Programme, were introduced with similar goals. ${ }^{17}$

More recent reviews (More care, less pathway; One Chance to Get It Right) have also identified shortcomings in the way PC services have developed and serve patients and those close to them. ${ }^{18,19}$ This has renewed enthusiasm to review current provision, refocus efforts, funding and care, and explore innovative approaches to manage and support patients with cancer.

\section{SPC guidelines}

Current literature is positive about earlier integration of SPC in cancer, whether or not the intent is cure. Earlier integration may provide improved QoL, increased survival, superior symptom management and less aggressive care towards the end of life. ${ }^{20-23}$ Such an approach does, however, rely on the appropriate referral of patients to SPC services.

No national guideline exists for SPC referral, as acknowledged in Commissioning Guidance for Specialist Palliative Care which recommends referral criteria that can be seen in figure $2 .{ }^{24}$ (Figure 2) Of four local UK NHS Trust policies, two advised an essential criteria as the presence of progressive, life-limiting or life-threatening illness. A common theme is that patient needs should be too complex for the 'non-SPC' team to manage. However, lack of national guidelines results in a referral based on colloquial opinion. Referral criteria are not without their problems; the use of checklist based criteria is often inappropriate, as individual situations are unique. On the other hand, a lack of specific referral criteria could potentially restrict access to SPC services. 
Clinical understanding of SPC has considerable variation. ${ }^{3}$ One study found that oncologists were more likely to make a referral to SPC at an early stage if they had clinical experience of SPC. This suggests that lack of education, training and experience about SPC could play a role in delaying referrals. ${ }^{25} \mathrm{~A}$ potential solution could be the use of an automated referral system where the patient's status or needs meet certain criteria, although this too could present problems through lack of individualisation.

\section{Quality of life assessments}

The mainstay of SPC is focus on QoL; the concept that care should be about quality and not quantity of life. Assessment of a patient's QoL may be made using validated tools such as the "Quality of life Functional Assessment of Chronic Illness Therapy-Spiritual Well-Being" (FACIT-Sp) or the "Functional Assessment of Cancer Therapy - Lung" (FACT-L) scales. ${ }^{26,27}$

QoL can be a measure of a patient's symptom burden, their ability to carry out daily tasks, the value they place on their life and their social, emotional and spiritual well-being. The FACIT-Sp scale (higher score, better QoL) demonstrated an increase in QoL for those who were subject to the earlier integration of SPC in a trial conducted by Zimmerman et al. ${ }^{21}$ There was a reduction in FACIT-Sp scores in the control group at all measured timepoints, compared to an increase in scores for the group subject to early integration. A statistically significant difference was noted at 4 months $(-3.95 \mathrm{vs}+2.46) .{ }^{21}$ Earlier SPC consisted of outpatient appointments, structured well-being assessments, routine follow-up (either in person or by telephone) and assessment by a multi-disciplinary team.

Temel et al. observed the effects of early SPC in patients with metastatic non-small-cell lung cancer using a novel Trial Outcome Index (TOI) utilising a summation of specific subscales 
within the FACT-L scale. ${ }^{20}$ An increased QoL was noted in the early SPC group after 12 weeks. The opposite was observed in the standard oncological care group. ${ }^{20}$ Two trials have thus shown statistically significant improvement in QoL following the earlier integration of SPC. However, data from these studies may not be reliably transferrable, as both were single centre studies in academic institutions. Furthermore, other studies have demonstrated mixed results ranging through no effect, delayed effect, positive effect for carers rather than patients, no effect on QoL but positive on secondary outcomes, to positive effect. ${ }^{28-34}$ Although results are varied, the evidence still suggests there is a benefit from exploring earlier SPC in patients with cancer.

\section{Symptom management}

SPC plays a significant role in symptom management, especially pain and loss of appetite, and patients with incurable disease experience a worsening picture of symptoms. ${ }^{35}$ Patients undergoing treatment experience symptoms related to disease and the side effects of treatment. SPC aims to manage both symptom burden of disease and side effects of any curative or palliative treatment.

Few trials specifically observe symptom intensity and improvement. ENABLE (Educate, Nurture, Advise, Before Life Ends) is a telephone based SPC service, where the secondary measure is symptom intensity, rated using the symptom impact subscale of the Quality of Life at End of Life scale. Results were not statistically significant. ${ }^{31-33}$ The ENABLE II Randomised Control Trial (RCT) demonstrated a trend in symptom improvement using the Edmonton Symptom Assessment Scale, allowing much greater detail in the score. ${ }^{33}$ The trend of reduced symptom intensity was not statistically significant. ${ }^{33}$ Temel et al. investigated mood (Hospital Anxiety and Depression Scale and Patient Health Questionnaire 9) and the 
results of this study can be seen in figure 3 where a statistically significant reduction in mood symptoms was noted in the early SPC intervention group, but new prescription rates for antidepressants were almost identical in both the standard care and early SPC group. ${ }^{20}$ (Figure 3) Symptom intensity demonstrates a trend to decrease with early SPC intervention and, with depressive symptoms specifically, show a statistically significant reduction..$^{20,31,33}$

\section{Effects on survival}

Survival is not a specific concern in SPC, but some literature demonstrates that earlier intervention leads to improvements in survival. Bakitas et al. measured patients' one-year survival following study enrolment. ${ }^{33} 63 \%$ of patients receiving earlier SPC achieved oneyear survival, in contrast to $48 \%$ of patients in the control group. ${ }^{33}$ Temel et al. investigated the effect of early access to SPC on patient-reported outcomes and end of life care and reported a significant difference in survival (median survival 11.6 months with early SPC; 8.9 months in the control group). ${ }^{20}$ The specific reasons for increased survival with early SPC intervention are not clear. Improved QoL and reduced depressive symptoms may have an impact on prognosis. ${ }^{36}$ SPC management of cancer treatment side effects could improve the ability to tolerate treatment. ${ }^{22}$

\section{End of life planning}

Earlier integration of SPC can facilitate better end of life (EoL) planning and, thus, a smoother transition from one phase of an illness to the next. Integration of SPC allows earlier conversations about advance care planning options such as Advance Decisions to Refuse Treatment, Do Not Attempt Cardiopulmonary Resuscitation, Lasting Power of Attorney and EoL preferences, such as preferred place of care and death. $81 \%$ of people have expressed a wish to die at home, yet Public Health England (PHE) recorded that $47 \%$ of deaths occurred 
in hospital. ${ }^{35-37}$ PHE noted that only $23 \%$ of deaths occurred in patients' own homes. ${ }^{37}$ This difference presents a clear argument for the lack of achievement of patient preferences. Early integration of SPC can contain the provision of home PC where such services exist, with increased odds of a home death $(+2.21) .^{38}$

In the study by Temel et al, $28 \%$ of the standard care cohort had advance care plans as compared to $53 \%$ in the early integration group. ${ }^{20}$ They also found the median length of hospice stay for standard care patients to be four days, compared to eleven days for those receiving early SPC. ${ }^{20}$ Introducing patients to hospice care at an earlier point in their illness may allow for better symptom management and increased QoL in later stages of disease. Differences exist in the nature of end of life care between the United States (US) and the United Kingdom (UK). In the US, the majority (75\%) of people die in institutions rather than at home; pain and other distress at the end of life is often undertreated as a result of funding mechanisms; and there is no national strategy for EoL care. ${ }^{39,40}$

EoL care in the UK has been found to be less 'aggressive'; i.e. patients are not subjected to unnecessary interventions, as they may be in the US. ${ }^{41}$ This could be attributed to recording of personal preferences about care and the subsequent withdrawal of aggressive cancer treatment. On the other hand, decreased symptom burden through better management could increase tolerance to cancer treatment and be a reason for increased survival seen with early SPC. $^{22,33}$ Admission rates to hospital have changed following earlier integration of SPC. Intensive Care Unit admissions, common in the US for PC patients, are seen to decrease by $10 \%$ (14\% versus $4 \%$ ), prolonged hospital admission ( $\geq 2$ weeks) decreased by $12 \%(20 \%$ versus $8 \%$ ) and emergency presentations in the last 30 days of life fell by $20 \%$ (68\% versus $48 \%)^{22}$ 
It would appear that earlier incorporation of SPC leads to a greater QoL in EoL care as a result of improved care planning, improved symptom control, a greater likelihood of death in the patient's preferred place, decreased hospital admission rates and less aggressive EoL treatments.

\section{Financial considerations}

The financial implications of SPC delivery are under close scrutiny. The National Institute for Health and Care Excellence (NICE) evaluates cost effectiveness through cost-utility. Benefits are assessed via quality-adjusted life years (QALYs) gained. Any effective treatment that costs the National Health Service (NHS) less than $£ 20000$ (\$31 000; €23 400) per QALY gained is perceived as cost effective. ${ }^{42}$ This is not to say, however, that all treatments are purely assessed on cost. ${ }^{42}$

There are significant differences in costs between early SPC and standard care. ${ }^{43}$ Following adjustment for the differences in survival days, a 33\% reduction in cost was noted with early SPC. Mean cost per day reduced from $\$ 212.80$ (£106.40; $€ 159.60)$ to $\$ 95.30$ (£47.65; $€ 71.48$ ), this is demonstrated in figure $4 .{ }^{44}$ (Figure 4) A Cochrane review established that, in all studies investigating cost the intervention groups (early SPC) led to cost reductions of $18 \%-35 \%$, yet only one trial was statistically significant. ${ }^{43,45}$ Greer et al. found evidence to the contrary though. They suggest that SPC is $\$ 11,260$ ( $£ 8,661 ; € 10,393)$ more expensive on average. $^{46}$ This leads to a complex problem for healthcare providers. Any increased cost requires justification and will be subject to close scrutiny, yet evidence suggests that QoL is greatly increased. There may be fewer hospital appointments, fewer emergency admissions or 
in-patient hospital stays, but increases in prescriptions for medication or care costs, for example.

\section{Barriers}

Evidence for the earlier integration of SPC appears to be favourable towards certain elements of early SPC provision but the data is inconsistently statistically significant, although does appear to demonstrate trends towards benefit. Despite this, there are no comprehensive plans for earlier integration of SPC in the UK. Perception is an obstacle. Understanding of SPC, by both patients and healthcare professionals, is poor. ${ }^{2,3}$ For successful implementation of early SPC, clinical staff require greater education and training as to what SPC is, what it can provide and how it can be accessed. This should be followed by patients being made aware that SPC is focused on supporting them and their families to achieve a better QoL. This takes time; time that, at present, healthcare staff do not possess. Unless this is addressed SPC, early or standard, will not be available to all those that could potentially benefit.

QoL increases in patients with cancer with early SPC but this has only been examined in a small number of tumour groups. NICE's QALY system is also difficult to apply to SPC as it does not take into account the value different people put on survival and the positive effect

the patient can have on others. ${ }^{47} \mathrm{SPC}$ is as much about supporting family members as it is about supporting the patient. A QALY metric cannot calculate for this and thus may underrepresent the benefits of SPC.

Greer et al. found SPC more expensive on average. ${ }^{46}$ Cancer Research UK suggests a figure for UK cancer deaths in 2014 to be 163,444 people. ${ }^{48}$ If 100,000 of this number could be eligible for early SPC, this would require an additional $\$ 1,126,000,000$ ( $£ 866,100,000$; 
$€ 1,039,300,000)$ in funding. The UK Clinical Research Collaboration found that, of just over $£ 2$ billion ( $\$ 3.32$ billion; $€ 2.4$ billion) spent on healthcare research in the UK in 2014 , only $0.16 \%$ was spent on end of life care. ${ }^{48}$ The National Cancer Research Institute Database shows that the proportion of cancer research funding spend on SPC and EoL care was consistently less than $0.7 \%$ over 12 years. ${ }^{44}$ Increasing provision, either by offering SPC to more people or offering it earlier will require money and resources that are not currently available. Offering SPC monitoring through out-patient appointments or home visits would require more clinical, nursing and administrative staff.

Disjointed communication between oncologists and SPC providers may represent another barrier, although communication between these specialties may be improving through the introduction of Acute Oncology Services in secondary care where relationships are strengthening. Oncologists are often at the front line of malignant disease management in the earlier stages of disease. Integration of oncology and SPC services may improve the delivery of high quality SPC. The traditional model of care in cancer is focused on oncology with other specialties, until it is apparent the patient is approaching the end of their life. An updated model which proposes the incorporation of SPC much earlier in the illness trajectory is demonstrated in figure 5, where colleagues would align their efforts from diagnosis through treatment or palliation, during decline, at end of life and into bereavement. (Figure 5)

Almost all identified barriers are amenable to remedy but, as with all healthcare provision, funding remains the main barrier to progress. The importance of the impact and effect of SPC must be considered further and evidenced through high powered RCTs with statistically significant results in favour of earlier integration. Funding these RCTs though is difficult, due to a lack of pharmacological innovation in this specialty, leading to a lack of commercial 
sponsorship potential. Until significant and positive trial data exists, increased funding may not be accessible and, as a result, improved provision of care, education, training and resources are not possible.

\section{Conclusions}

By conducting this literature review we have learned of the difficulties surrounding the nomenclature of Specialist Palliative care, that there is a misunderstanding of the specialty amongst the healthcare profession and the general public and, in response to this, have identified and outlined a recent increase in programmes designed to educate healthcare professionals on the topic. A lack of guidelines in the UK health system on when to refer a patient to SPC services has been highlighted. However, the implementation of guidelines may not be effective due to the unique situation of each patient, inviting further research in this area. Looking to whether earlier integration of SPC would be beneficial, there is evidence in favour of earlier integration for cancer patients and those close to them that may improve QoL, reduce symptom burden, extend life and, at the end of life, reduce the need for unnecessary intervention and, often, aggressive care. These are all positive outcomes.

Yet, as with all innovations in healthcare, barriers exist. Lack of awareness and education of clinicians, teamed with the lack of agreed referral guidelines hinders access to SPC. Delays in treatment and care exist because of a lack of effective communication between clinicians of different specialties and across organisational boundaries, and can lead to a poorer patient experience. This can be corrected, but an overarching theme seems to be the lack of funding and resources to address these issues. 
SPC requires further investigation by well-designed RCTs. If results show significant advantages to earlier integration of SPC that are deemed to be worth increased expenditure, then earlier implementation in a cancer patient's journey would be highly recommended. 


\section{References}

1. Buck, PS. My Several Worlds. New York: Day, 1957:337.

2. Maciasz R, Arnold R, Chu E, et al. Does it matter what you call it? A randomized trial of language used to describe palliative care services. Support Care Cancer 2013;21(12):3411-9.

3. McInturff, B, Harrington, L. Public Opinion Strategies. Presentation of 2011 Research on Palliative Care. New York: Center to Advance Palliative Care, American Cancer Society Cancer Action Network, 2011: June 13.

4. Hinton J. Which patients with terminal cancer are admitted from home care? Palliat Med 1994;8(3):197-210.

5. WHO. World Health Organization. Cancer Fact Sheet. 2017 [cited 2017 Feb 26]. http://www.who.int/mediacentre/factsheets/fs297/en/ (accessed 16 November 2017).

6. General Medical Council. Treatment and care towards the end of life: good practice in decision-making. London: General Medical Council, 2010.

7. El-Jawahri A, Greer JA, Temel JS. Does palliative care improve outcomes for patients with incurable illness? A review of the evidence. J Support Oncol 2011;9(3):87-94.

8. National Cancer Institute: Dictionary of Cancer Terms [cited 2017 Mar 5]. https://www.cancer.gov/publications/dictionaries/cancer-terms (accessed 16 November 2017).

9. General Medical Council. Good Medical Practice: End of Life Care. London: General Medical Council, 2010.

10. Baines, M. From pioneer days to implementation: lessons to be learnt. Eur J Palliat Care 2011;18(5):223-7. 
11. Johnson MJ, Eva GE, Booth S. Palliative medicine and symptom control. In: Kumar P, Clark ML (eds), Clinical Medicine, 8th edn. Philadelphia: WB Saunders Company, 2012 (485-92).

12. Department of Health. A High Quality Workforce: NHS Next Stage Review. London: Department of Health, 2008.

13. Gold Standards Framework: Welcome to Gold Standards Framework. Available at: http://www.goldstandardsframework.org.uk [accessed 2 May 2018]

14. BMJ Careers: Macmillan GP facilitators. Available at: http://careers.bmj.com/careers/advice/Macmillan_GP_facilitators

15. Thomas K. Caring for the dying at home. Oxford: Radcliffe Medical, 2003.

16. Department of Health. End of Life Care Strategy: promoting high quality care for adults at the end of their life. London: Department of Health, 2008.

17. NHS England. Transforming End of Life Care in Acute Hospitals: the Route to Success 'How To’ Guide. Leicester: NHS England, 2015.

18. Neuberger J. More care, less pathway: a review of the Liverpool Care Pathway. London: Department of Health, 2013.

19. Leadership Alliance for the Care of Dying People. One chance to get it right: improving people's experience of care in the last few days and hours of life. London: Leadership Alliance for the Care of Dying People, 2014.

20. Temel JS, Greer JA, Muzikansky A, et al. Early palliative care for patients with Metastatic Non-Small-Cell lung cancer. New Engl J Med 2010;363(8):733-42.

21. Zimmermann C, Swami N, Krzyzanowska M, et al. Early palliative care for patients with advanced cancer: A cluster-randomised controlled trial. Lancet 2014;383(9930):1721-30.

22. Hui D, Bruera E. Integrating palliative care into the trajectory of cancer care. Nat Rev Clin Oncol 2015;13(3):159-71. 
23. Hui D, Kim SH, Roquemore $\mathrm{J}$, et al. Impact of timing and setting of palliative care referral on quality of end-of-life care in cancer patients. Cancer 2014;120(11):1743-9.

24. Commissioning Guidance for Specialist Palliative Care: Helping to deliver commissioning objectives, December 2012. Guidance document published collaboratively with the Association for Palliative Medicine of Great Britain and Ireland, Consultant Nurse in Palliative Care Reference Group, Marie Curie Cancer Care, National Council for Palliative Care, and Palliative Care Section of the Royal Society of Medicine, London, UK.

25. Wentlandt K, Krzyzanowska M, Swami N, et al. Referral Practices of Oncologists to Specialized Palliative Care. J Clin Oncol 2012;30(35):4380-6.

26. Haugan G. The FACIT-Sp spiritual well-being scale: an investigation of the dimensionality, reliability and construct validity in a cognitively intact nursing home population. Scand J Caring Sci 2015; 29(1):152-64.

27. Cella DF, BonoLloyd SR, Tulsky DS, Kaplan E, Bonomi P. Reliability and validity of the Functional Assessment of Cancer Therapy-Lung (FACT-L) quality of life instrument. Lung Cancer 1995;12(3):199-220.

28. Groenvold M, Petersen MA, Damkier A, et al. Randomised clinical trial of early specialist palliative care plus standard care versus standard care alone in patients with advanced cancer: The Danish Palliative Care Trial. Palliat Med 2017 [Epub ahead of print].

29. McCorkle R, Jeon S, Ercolano E, et al. An Advanced Practice Nurse Coordinated Multidisciplinary Intervention for Patients with Late-Stage Cancer: A Cluster Randomized Trial. J Palliat Med 2015;18:962-9. 
30. Temel JS, Greer JA, El-Jawahri A, et al. Effects of Early Integrated Palliative Care in Patients With Lung and GI Cancer: A Randomized Clinical Trial. J Clin Oncol 2017;35:834-41.

31. Bakitas M, Tosteson T, Li Z, et al. Early Versus Delayed Initiation of Concurrent Palliative Oncology Care: Patient Outcomes in the ENABLE III Randomized Controlled Trial. J Clin Oncol 2015;33(13):1438-45.

32. Dionne-Odom JN, Azuero A, Lyons KD, et al. Benefits of Early Versus Delayed Palliative Care to Informal Family Caregivers of Patients With Advanced Cancer: Outcomes From the ENABLE III Randomized Controlled Trial. J Clin Oncol 2015;33:1446-52.

33. Bakitas M, Lyons K, Hegel M, et al. Effects of a Palliative Care Intervention on Clinical Outcomes in Patients With Advanced Cancer. The Project ENABLE II randomised control trial. JAMA 2009;302(7):741.

34. Maltoni M, Scarpi E, Dall'Agata M, et al. Early Palliative Care Italian Study Group (EPCISG). Systematic versus on-demand early palliative care: results from a multicentre, randomised clinical trial. Eur J Cancer 2016;65:61-8.

35. Office for National Statistics, National Survey of Bereaved People (VOICES): England, 2015 [cited 2017 Mar 15]. Available from: https://www.ons.gov.uk/peoplepopulationandcommunity/healthandsocialcare/healthcares ystem/bulletins/nationalsurveyofbereavedpeoplevoices/england2015\#preferences-andchoice-at-the-end-of-life

36. Lutz S, Norrell R, Bertucio C, et al. Symptom Frequency and Severity in Patients with Metastatic or Locally Recurrent Lung Cancer: A Prospective Study Using the Lung Cancer Symptom Scale in a Community Hospital. J Palliat Med 2001;4(2):157-65. 
37. Maione P, Perrone F, Gallo C, et al. Pretreatment Quality of Life and Functional Status Assessment Significantly Predict Survival of Elderly Patients With Advanced Non SmallCell Lung Cancer Receiving Chemotherapy: A Prognostic Analysis of the Multicenter Italian Lung Cancer in the Elderly Study. J Clin Oncol 2005;23(28):6865-72.

38. Basch E, Deal AM, Dueck AC, et al. Survival Results of a Trial Assessing PatientReported Outcomes for Symptom Monitoring During Routine Cancer Treatment. JAMA 2017;318(2):197-8.

39. Basch E, Deal AM, Kris MG, et al. Symptom Monitoring With Patient-Reported Outcomes During Routine Cancer Treatment: A Randomized Controlled Trial. J Clin Oncol 2016;34(6):557-65.

40. Office for National Statistics. National Survey of Bereaved People (VOICES). London: Office for National Statistics, 2015.

41. Public Health England. National End of Life Care Intelligence Network. Number and proportion of deaths by place of occurrence. London: Public Health England, 2015.

42. National Institute for health and Care Excellence. NICE Guidance: Judging whether public health interventions offer value for money. London: NICE, 2013

43. Brumley R, Enguidanos S, Jamison P, et al. Increased Satisfaction with Care and Lower Costs: Results of a Randomized Trial of In-Home Palliative Care. J Am Geriatr Soc 2007;55(7):993-1000.

44. Marie Curie. The hidden challenges of palliative cancer care, 2016. London: Marie Curie, 2016

45. Gomes B, Calanzani N, Curiale V, McCrone P, Higginson I. Effectiveness and costeffectiveness of home palliative care services for adults with advanced illness and their caregivers. Cochrane Database Syst Review 2013 Jun 6;(6):CD007760. 
46. Greer J, Tramontano A, McMahon P, et al. Cost Analysis of a Randomized Trial of Early Palliative Care in Patients with Metastatic Non Small-Cell Lung Cancer. J Palliat Med 2016;19(8):842-8.

47. Normand C. Measuring Outcomes in Palliative Care: Limitations of QALYs and the Road to PalYs. J Pain Symptom Manage 2009;38(1):27-31.

48. UK Clinical Research Collaboration. UK Health Research Analysis 2014. London: Medical Research Council, 2014.

49. Hui D, De La Cruz M, Mori M, et al. Concepts and definitions for 'supportive care,' 'best supportive care,' 'palliative care,' and 'hospice care' in the published literature, dictionaries, and textbooks. Support Care Cancer 2012;21(3):659-85.

50. EPEC-O, education in palliative and end-of-life care for oncology [electronic resource]. U.S. Department of Health and Human Services, National Institutes of Health, National Cancer Institute, American Society of Clinical Oncology, Hospice and Palliative Nurses Association. U.S. Department of Health and Human Services, National Institutes of Health, National Cancer Institute, 2006. 


\section{Figures}

Figure 1: A timeline demonstrating the differences in care plotted against disease progression resulting in death. Adapted from Hui, D et al. ${ }^{49}$

Figure 2: Referral criteria to access SPC. Adapted from APM Commissioning guidance for specialist care. ${ }^{24}$

Figure 3: Mood assessment scores in standard care compared to early palliative care. Adapted from Temel et al. ${ }^{20}$

Figure 4: Mean cost of care in standard care compared to early palliative care. Adapted from Brumley et al. $^{43}$

Figure 5: Proposed involvement of healthcare disciplines, plotted against disease progression. Adapted from the National Cancer Institute. ${ }^{50}$ 
Integration of early supportive and palliative care in a patient's journey with cancer Word count: $\quad$ Abstract: 166; Body text: 3401

Author contributions: The authors confirm that all authors listed meet all four of the criteria recommended by the ICMJE. 
Integration of early supportive and palliative care in a patient's journey with cancer: a review

\begin{abstract}
Specialist Palliative Care aims to effectively support the quality of life of patients and those close to them through progressive, life-limiting disease. Quality of life, an individual concept, requires a personalised approach to support and maintain it. Primarily achieved through the management of symptoms, both physical and psychological, alongside social and spiritual support, this approach is of the utmost importance to patients with advanced malignancy.
\end{abstract}

Several randomised, controlled trials suggest earlier provision of specialist palliative care may increase quality of life, improve symptoms and facilitate considered end of life care planning. This appears beneficial; however, evidence is mixed about the effectiveness of early specialist palliative care and its potential benefits. Results, therefore, should be interpreted with caution.

In reviewing the literature, it is clear that implementing early specialist palliative care is fraught with obstacles and requires increased resources and funding. Until the benefits and cost implications for such provision are better understood, it will not be accessible to all that may have potential to benefit. 


\section{Introduction}

"The test of a civilisation is in the way that it cares for its helpless members".

$(\text { Buck, 1954) })^{1}$

One group of society's most "helpless members" includes those burdened with advanced disease and whose illness is not amenable to cure. This group is wide and disparate but includes patients diagnosed with malignant disease, whose illness journey may be short or can stretch to many years. No matter the course or timescale, the needs of cancer patients can be great and the quality of life (QoL) they experience can depend on the support they receive.

Popular opinion appears to be that palliative care (PC) is associated with death. Patients associate supportive care with psychological and social support; yet associate PC with endof-life care. $^{2}$ While partially true, palliation of symptoms is also appropriate throughout potentially curative treatment. This misperception may have a negative impact on the involvement of Specialist Palliative Care services (SPC) in patients' care. Physicians, nurses and other allied healthcare professionals also struggle with such differences in interpretation, and referrals to SPC can be hampered by this. ${ }^{3}$ This suggests a need for "re-branding" and a better "marketing" of SPC.

SPC is a distinct medical specialty, as opposed to generalist PC that is provided, often to a very high standard, by all doctors and healthcare professionals, but most notably by General Practitioners and other community-based professionals. ${ }^{4}$

To palliate originates from the Latin pallium, meaning "to cloak". SPC philosophy holds true 
to this; to cloak the symptoms and problems of those with life-limiting illness. The fact that SPC originated in cancer care and this is still its main focus is unsurprising, as one in six deaths globally is the result of cancer and, at least at the specialty's inception, treatments available for this group of patients were few. ${ }^{5}$

While SPC in the management of cancer has developed to provide expert input at the end of life, it has recently been recognised that SPC may be applicable much earlier: at diagnosis, through potentially curative treatment and at appropriate points throughout the course of malignant disease. ${ }^{6}$ Effective PC aims to achieve the best QoL possible and it seems obvious that this would be beneficial; so would earlier integration of SPC be beneficial to patients, and those close to them, in their journey with cancer? ${ }^{7}$

To explore this, we first investigate the meaning of Specialist Palliative Care and the other names given to this discipline. We then look into what drives progress in the field of SPC and what guidelines are available to healthcare professionals. Following this, we look at what difference, if any, can be made by incorporating palliative care earlier into a patient's journey by examining the research based around improving quality of life, symptom management, survival and end of life planning. We conclude this article by considering potential barriers to earlier integration and what conclusions can be drawn from our research.

\section{Specialist Palliative Care / Supportive and Palliative Care}

SPC and Supportive and Palliative Care are in essence the same and, indeed, the National Cancer Institute attributes the same definition to both. ${ }^{8}$ The existence of both terms could be understood in Figure 1, where it suggests how Supportive Care and Palliative Care could be applicable at different points in a patient's progressively deteriorating condition (Figure 1). 
SPC, according to the General Medical Council, is "the holistic care of persons with advanced, progressive, incurable illness", specifically aimed towards managing patients' symptoms and side effects of treatment. ${ }^{9}$ Management is based on the model of 'total pain', where not only physical symptoms but also psychological, social and spiritual issues are addressed $^{10}$ and support for those close to the patient is equally important. This may appear to be a 'non-medical' approach, but if the healthcare team doesn't acknowledge, explore and address such issues, medical actions and interventions to address physical issues may be rendered futile. Additionally, advance care planning should be initiated early in the patient journey and be a continuous process. ${ }^{11}$ It is clear, then, that in order to achieve 'total pain' management, a multidisciplinary team of doctors, nurses, allied health professionals and those who represent religious and other beliefs are required.

\section{Drivers}

How those approaching the end of their lives are cared for is an indicator of how we care for all sick and vulnerable people in our society. ${ }^{12}$ The last decade has seen an increasing awareness and interest in palliative and end of life care and recognition of the importance of 'generic' PC.

The Gold Standards Framework and Macmillan GP Facilitator programmes aimed to improve the PC of patients in the community and improve access to educational opportunities for primary care professionals. ${ }^{12,13}$ National initiatives, including A High Quality Workforce: NHS Next Stage Review and the End of Life Care Strategy identified the importance of further development of PC in the UK. ${ }^{14-16}$ Secondary care based initiatives, such as the 
Routes to Success in End of Life Care and the Transforming End of Life Care in Acute Hospitals Programme, were introduced with similar goals. ${ }^{17}$

More recent reviews (More care, less pathway; One Chance to Get It Right) have also identified shortcomings in the way PC services have developed and serve patients and those close to them. ${ }^{18,19}$ This has renewed enthusiasm to review current provision, refocus efforts, funding and care, and explore innovative approaches to manage and support patients with cancer.

\section{SPC guidelines}

Current literature is positive about earlier integration of SPC in cancer, whether or not the intent is cure. Earlier integration may provide improved QoL, increased survival, superior symptom management and less aggressive care towards the end of life. ${ }^{20-23}$ Such an approach does, however, rely on the appropriate referral of patients to SPC services.

No national guideline exists for SPC referral, as acknowledged in Commissioning Guidance for Specialist Palliative Care which recommends referral criteria that can be seen in figure $2 .{ }^{24}$ (Figure 2) Of four local UK NHS Trust policies, two advised an essential criteria as the presence of progressive, life-limiting or life-threatening illness. A common theme is that patient needs should be too complex for the 'non-SPC' team to manage. However, lack of national guidelines results in a referral based on colloquial opinion. Referral criteria are not without their problems; the use of checklist based criteria is often inappropriate, as individual situations are unique. On the other hand, a lack of specific referral criteria could potentially restrict access to SPC services. 
Clinical understanding of SPC has considerable variation. ${ }^{3}$ One study found that oncologists were more likely to make a referral to SPC at an early stage if they had clinical experience of SPC. This suggests that lack of education, training and experience about SPC could play a role in delaying referrals. ${ }^{25} \mathrm{~A}$ potential solution could be the use of an automated referral system where the patient's status or needs meet certain criteria, although this too could present problems through lack of individualisation.

\section{Quality of life assessments}

The mainstay of SPC is focus on QoL; the concept that care should be about quality and not quantity of life. Assessment of a patient's QoL may be made using validated tools such as the "Quality of life Functional Assessment of Chronic Illness Therapy-Spiritual Well-Being" (FACIT-Sp) or the "Functional Assessment of Cancer Therapy - Lung" (FACT-L) scales. ${ }^{26,27}$

QoL can be a measure of a patient's symptom burden, their ability to carry out daily tasks, the value they place on their life and their social, emotional and spiritual well-being. The FACIT-Sp scale (higher score, better QoL) demonstrated an increase in QoL for those who were subject to the earlier integration of SPC in a trial conducted by Zimmerman et al. ${ }^{21}$ There was a reduction in FACIT-Sp scores in the control group at all measured timepoints, compared to an increase in scores for the group subject to early integration. A statistically significant difference was noted at 4 months $(-3.95 \mathrm{vs}+2.46) .{ }^{21}$ Earlier SPC consisted of outpatient appointments, structured well-being assessments, routine follow-up (either in person or by telephone) and assessment by a multi-disciplinary team.

Temel et al. observed the effects of early SPC in patients with metastatic non-small-cell lung cancer using a novel Trial Outcome Index (TOI) utilising a summation of specific subscales 
within the FACT-L scale. ${ }^{20}$ An increased QoL was noted in the early SPC group after 12 weeks. The opposite was observed in the standard oncological care group. ${ }^{20}$ Two trials have thus shown statistically significant improvement in QoL following the earlier integration of SPC. However, data from these studies may not be reliably transferrable, as both were single centre studies in academic institutions. Furthermore, other studies have demonstrated mixed results ranging through no effect, delayed effect, positive effect for carers rather than patients, no effect on QoL but positive on secondary outcomes, to positive effect. ${ }^{28-34}$ Although results are varied, the evidence still suggests there is a benefit from exploring earlier SPC in patients with cancer.

\section{Symptom management}

SPC plays a significant role in symptom management, especially pain and loss of appetite, and patients with incurable disease experience a worsening picture of symptoms. ${ }^{35}$ Patients undergoing treatment experience symptoms related to disease and the side effects of treatment. SPC aims to manage both symptom burden of disease and side effects of any curative or palliative treatment.

Few trials specifically observe symptom intensity and improvement. ENABLE (Educate, Nurture, Advise, Before Life Ends) is a telephone based SPC service, where the secondary measure is symptom intensity, rated using the symptom impact subscale of the Quality of Life at End of Life scale. Results were not statistically significant. ${ }^{31-33}$ The ENABLE II Randomised Control Trial (RCT) demonstrated a trend in symptom improvement using the Edmonton Symptom Assessment Scale, allowing much greater detail in the score. ${ }^{33}$ The trend of reduced symptom intensity was not statistically significant. ${ }^{33}$ Temel et al. investigated mood (Hospital Anxiety and Depression Scale and Patient Health Questionnaire 9) and the 
results of this study can be seen in figure 3 where a statistically significant reduction in mood symptoms was noted in the early SPC intervention group, but new prescription rates for antidepressants were almost identical in both the standard care and early SPC group. ${ }^{20}$ (Figure 3) Symptom intensity demonstrates a trend to decrease with early SPC intervention and, with depressive symptoms specifically, show a statistically significant reduction..$^{20,31,33}$

\section{Effects on survival}

Survival is not a specific concern in SPC, but some literature demonstrates that earlier intervention leads to improvements in survival. Bakitas et al. measured patients' one-year survival following study enrolment. ${ }^{33} 63 \%$ of patients receiving earlier SPC achieved oneyear survival, in contrast to $48 \%$ of patients in the control group. ${ }^{33}$ Temel et al. investigated the effect of early access to SPC on patient-reported outcomes and end of life care and reported a significant difference in survival (median survival 11.6 months with early SPC; 8.9 months in the control group). ${ }^{20}$ The specific reasons for increased survival with early SPC intervention are not clear. Improved QoL and reduced depressive symptoms may have an impact on prognosis. ${ }^{36}$ SPC management of cancer treatment side effects could improve the ability to tolerate treatment. ${ }^{22}$

\section{End of life planning}

Earlier integration of SPC can facilitate better end of life (EoL) planning and, thus, a smoother transition from one phase of an illness to the next. Integration of SPC allows earlier conversations about advance care planning options such as Advance Decisions to Refuse Treatment, Do Not Attempt Cardiopulmonary Resuscitation, Lasting Power of Attorney and EoL preferences, such as preferred place of care and death. $81 \%$ of people have expressed a wish to die at home, yet Public Health England (PHE) recorded that $47 \%$ of deaths occurred 
in hospital. ${ }^{35-37}$ PHE noted that only $23 \%$ of deaths occurred in patients' own homes. ${ }^{37}$ This difference presents a clear argument for the lack of achievement of patient preferences. Early integration of SPC can contain the provision of home PC where such services exist, with increased odds of a home death $(+2.21) .^{38}$

In the study by Temel et al, $28 \%$ of the standard care cohort had advance care plans as compared to $53 \%$ in the early integration group. ${ }^{20}$ They also found the median length of hospice stay for standard care patients to be four days, compared to eleven days for those receiving early SPC. ${ }^{20}$ Introducing patients to hospice care at an earlier point in their illness may allow for better symptom management and increased QoL in later stages of disease. Differences exist in the nature of end of life care between the United States (US) and the United Kingdom (UK). In the US, the majority (75\%) of people die in institutions rather than at home; pain and other distress at the end of life is often undertreated as a result of funding mechanisms; and there is no national strategy for EoL care. ${ }^{39,40}$

EoL care in the UK has been found to be less 'aggressive'; i.e. patients are not subjected to unnecessary interventions, as they may be in the US. ${ }^{41}$ This could be attributed to recording of personal preferences about care and the subsequent withdrawal of aggressive cancer treatment. On the other hand, decreased symptom burden through better management could increase tolerance to cancer treatment and be a reason for increased survival seen with early SPC. $^{22,33}$ Admission rates to hospital have changed following earlier integration of SPC. Intensive Care Unit admissions, common in the US for PC patients, are seen to decrease by $10 \%$ (14\% versus $4 \%$ ), prolonged hospital admission ( $\geq 2$ weeks) decreased by $12 \%(20 \%$ versus $8 \%$ ) and emergency presentations in the last 30 days of life fell by $20 \%$ (68\% versus $48 \%)^{22}$ 
It would appear that earlier incorporation of SPC leads to a greater QoL in EoL care as a result of improved care planning, improved symptom control, a greater likelihood of death in the patient's preferred place, decreased hospital admission rates and less aggressive EoL treatments.

\section{Financial considerations}

The financial implications of SPC delivery are under close scrutiny. The National Institute for Health and Care Excellence (NICE) evaluates cost effectiveness through cost-utility. Benefits are assessed via quality-adjusted life years (QALYs) gained. Any effective treatment that costs the National Health Service (NHS) less than $£ 20000$ (\$31 000; €23 400) per QALY gained is perceived as cost effective. ${ }^{42}$ This is not to say, however, that all treatments are purely assessed on cost. ${ }^{42}$

There are significant differences in costs between early SPC and standard care. ${ }^{43}$ Following adjustment for the differences in survival days, a 33\% reduction in cost was noted with early SPC. Mean cost per day reduced from $\$ 212.80$ (£106.40; $€ 159.60)$ to $\$ 95.30$ (£47.65; $€ 71.48$ ), this is demonstrated in figure $4 .{ }^{44}$ (Figure 4) A Cochrane review established that, in all studies investigating cost the intervention groups (early SPC) led to cost reductions of $18 \%-35 \%$, yet only one trial was statistically significant. ${ }^{43,45}$ Greer et al. found evidence to the contrary though. They suggest that SPC is $\$ 11,260$ ( $£ 8,661 ; € 10,393)$ more expensive on average. $^{46}$ This leads to a complex problem for healthcare providers. Any increased cost requires justification and will be subject to close scrutiny, yet evidence suggests that QoL is greatly increased. There may be fewer hospital appointments, fewer emergency admissions or 
in-patient hospital stays, but increases in prescriptions for medication or care costs, for example.

\section{Barriers}

Evidence for the earlier integration of SPC appears to be favourable towards certain elements of early SPC provision but the data is inconsistently statistically significant, although does appear to demonstrate trends towards benefit. Despite this, there are no comprehensive plans for earlier integration of SPC in the UK. Perception is an obstacle. Understanding of SPC, by both patients and healthcare professionals, is poor. ${ }^{2,3}$ For successful implementation of early SPC, clinical staff require greater education and training as to what SPC is, what it can provide and how it can be accessed. This should be followed by patients being made aware that SPC is focused on supporting them and their families to achieve a better QoL. This takes time; time that, at present, healthcare staff do not possess. Unless this is addressed SPC, early or standard, will not be available to all those that could potentially benefit.

QoL increases in patients with cancer with early SPC but this has only been examined in a small number of tumour groups. NICE's QALY system is also difficult to apply to SPC as it does not take into account the value different people put on survival and the positive effect

the patient can have on others. ${ }^{47} \mathrm{SPC}$ is as much about supporting family members as it is about supporting the patient. A QALY metric cannot calculate for this and thus may underrepresent the benefits of SPC.

Greer et al. found SPC more expensive on average. ${ }^{46}$ Cancer Research UK suggests a figure for UK cancer deaths in 2014 to be 163,444 people. ${ }^{48}$ If 100,000 of this number could be eligible for early SPC, this would require an additional $\$ 1,126,000,000$ ( $£ 866,100,000$; 
$€ 1,039,300,000)$ in funding. The UK Clinical Research Collaboration found that, of just over $£ 2$ billion ( $\$ 3.32$ billion; $€ 2.4$ billion) spent on healthcare research in the UK in 2014 , only $0.16 \%$ was spent on end of life care. ${ }^{48}$ The National Cancer Research Institute Database shows that the proportion of cancer research funding spend on SPC and EoL care was consistently less than $0.7 \%$ over 12 years. ${ }^{44}$ Increasing provision, either by offering SPC to more people or offering it earlier will require money and resources that are not currently available. Offering SPC monitoring through out-patient appointments or home visits would require more clinical, nursing and administrative staff.

Disjointed communication between oncologists and SPC providers may represent another barrier, although communication between these specialties may be improving through the introduction of Acute Oncology Services in secondary care where relationships are strengthening. Oncologists are often at the front line of malignant disease management in the earlier stages of disease. Integration of oncology and SPC services may improve the delivery of high quality SPC. The traditional model of care in cancer is focused on oncology with other specialties, until it is apparent the patient is approaching the end of their life. An updated model which proposes the incorporation of SPC much earlier in the illness trajectory is demonstrated in figure 5, where colleagues would align their efforts from diagnosis through treatment or palliation, during decline, at end of life and into bereavement. (Figure 5)

Almost all identified barriers are amenable to remedy but, as with all healthcare provision, funding remains the main barrier to progress. The importance of the impact and effect of SPC must be considered further and evidenced through high powered RCTs with statistically significant results in favour of earlier integration. Funding these RCTs though is difficult, due to a lack of pharmacological innovation in this specialty, leading to a lack of commercial 
sponsorship potential. Until significant and positive trial data exists, increased funding may not be accessible and, as a result, improved provision of care, education, training and resources are not possible.

\section{Conclusions}

By conducting this literature review we have learned of the difficulties surrounding the nomenclature of Specialist Palliative care, that there is a misunderstanding of the specialty amongst the healthcare profession and the general public and, in response to this, have identified and outlined a recent increase in programmes designed to educate healthcare professionals on the topic. A lack of guidelines in the UK health system on when to refer a patient to SPC services has been highlighted. However, the implementation of guidelines may not be effective due to the unique situation of each patient, inviting further research in this area. Looking to whether earlier integration of SPC would be beneficial, there is evidence in favour of earlier integration for cancer patients and those close to them that may improve QoL, reduce symptom burden, extend life and, at the end of life, reduce the need for unnecessary intervention and, often, aggressive care. These are all positive outcomes.

Yet, as with all innovations in healthcare, barriers exist. Lack of awareness and education of clinicians, teamed with the lack of agreed referral guidelines hinders access to SPC. Delays in treatment and care exist because of a lack of effective communication between clinicians of different specialties and across organisational boundaries, and can lead to a poorer patient experience. This can be corrected, but an overarching theme seems to be the lack of funding and resources to address these issues. 
SPC requires further investigation by well-designed RCTs. If results show significant advantages to earlier integration of SPC that are deemed to be worth increased expenditure, then earlier implementation in a cancer patient's journey would be highly recommended. 


\section{References}

1. Buck, PS. My Several Worlds. New York: Day, 1957:337.

2. Maciasz R, Arnold R, Chu E, et al. Does it matter what you call it? A randomized trial of language used to describe palliative care services. Support Care Cancer 2013;21(12):3411-9.

3. McInturff, B, Harrington, L. Public Opinion Strategies. Presentation of 2011 Research on Palliative Care. New York: Center to Advance Palliative Care, American Cancer Society Cancer Action Network, 2011: June 13.

4. Hinton J. Which patients with terminal cancer are admitted from home care? Palliat Med 1994;8(3):197-210.

5. WHO. World Health Organization. Cancer Fact Sheet. 2017 [cited 2017 Feb 26]. http://www.who.int/mediacentre/factsheets/fs297/en/ (accessed 16 November 2017).

6. General Medical Council. Treatment and care towards the end of life: good practice in decision-making. London: General Medical Council, 2010.

7. El-Jawahri A, Greer JA, Temel JS. Does palliative care improve outcomes for patients with incurable illness? A review of the evidence. J Support Oncol 2011;9(3):87-94.

8. National Cancer Institute: Dictionary of Cancer Terms [cited 2017 Mar 5]. https://www.cancer.gov/publications/dictionaries/cancer-terms (accessed 16 November 2017).

9. General Medical Council. Good Medical Practice: End of Life Care. London: General Medical Council, 2010.

10. Baines, M. From pioneer days to implementation: lessons to be learnt. Eur J Palliat Care 2011;18(5):223-7. 
11. Johnson MJ, Eva GE, Booth S. Palliative medicine and symptom control. In: Kumar P, Clark ML (eds), Clinical Medicine, 8th edn. Philadelphia: WB Saunders Company, 2012 (485-92).

12. Department of Health. A High Quality Workforce: NHS Next Stage Review. London: Department of Health, 2008.

13. Gold Standards Framework: Welcome to Gold Standards Framework. Available at: http://www.goldstandardsframework.org.uk [accessed 2 May 2018]

14. BMJ Careers: Macmillan GP facilitators. Available at: http://careers.bmj.com/careers/advice/Macmillan_GP_facilitators

15. Thomas K. Caring for the dying at home. Oxford: Radcliffe Medical, 2003.

16. Department of Health. End of Life Care Strategy: promoting high quality care for adults at the end of their life. London: Department of Health, 2008.

17. NHS England. Transforming End of Life Care in Acute Hospitals: the Route to Success 'How To’ Guide. Leicester: NHS England, 2015.

18. Neuberger J. More care, less pathway: a review of the Liverpool Care Pathway. London: Department of Health, 2013.

19. Leadership Alliance for the Care of Dying People. One chance to get it right: improving people's experience of care in the last few days and hours of life. London: Leadership Alliance for the Care of Dying People, 2014.

20. Temel JS, Greer JA, Muzikansky A, et al. Early palliative care for patients with Metastatic Non-Small-Cell lung cancer. New Engl J Med 2010;363(8):733-42.

21. Zimmermann C, Swami N, Krzyzanowska M, et al. Early palliative care for patients with advanced cancer: A cluster-randomised controlled trial. Lancet 2014;383(9930):1721-30.

22. Hui D, Bruera E. Integrating palliative care into the trajectory of cancer care. Nat Rev Clin Oncol 2015;13(3):159-71. 
23. Hui D, Kim SH, Roquemore $\mathrm{J}$, et al. Impact of timing and setting of palliative care referral on quality of end-of-life care in cancer patients. Cancer 2014;120(11):1743-9.

24. Commissioning Guidance for Specialist Palliative Care: Helping to deliver commissioning objectives, December 2012. Guidance document published collaboratively with the Association for Palliative Medicine of Great Britain and Ireland, Consultant Nurse in Palliative Care Reference Group, Marie Curie Cancer Care, National Council for Palliative Care, and Palliative Care Section of the Royal Society of Medicine, London, UK.

25. Wentlandt K, Krzyzanowska M, Swami N, et al. Referral Practices of Oncologists to Specialized Palliative Care. J Clin Oncol 2012;30(35):4380-6.

26. Haugan G. The FACIT-Sp spiritual well-being scale: an investigation of the dimensionality, reliability and construct validity in a cognitively intact nursing home population. Scand J Caring Sci 2015; 29(1):152-64.

27. Cella DF, BonoLloyd SR, Tulsky DS, Kaplan E, Bonomi P. Reliability and validity of the Functional Assessment of Cancer Therapy-Lung (FACT-L) quality of life instrument. Lung Cancer 1995;12(3):199-220.

28. Groenvold M, Petersen MA, Damkier A, et al. Randomised clinical trial of early specialist palliative care plus standard care versus standard care alone in patients with advanced cancer: The Danish Palliative Care Trial. Palliat Med 2017 [Epub ahead of print].

29. McCorkle R, Jeon S, Ercolano E, et al. An Advanced Practice Nurse Coordinated Multidisciplinary Intervention for Patients with Late-Stage Cancer: A Cluster Randomized Trial. J Palliat Med 2015;18:962-9. 
30. Temel JS, Greer JA, El-Jawahri A, et al. Effects of Early Integrated Palliative Care in Patients With Lung and GI Cancer: A Randomized Clinical Trial. J Clin Oncol 2017;35:834-41.

31. Bakitas M, Tosteson T, Li Z, et al. Early Versus Delayed Initiation of Concurrent Palliative Oncology Care: Patient Outcomes in the ENABLE III Randomized Controlled Trial. J Clin Oncol 2015;33(13):1438-45.

32. Dionne-Odom JN, Azuero A, Lyons KD, et al. Benefits of Early Versus Delayed Palliative Care to Informal Family Caregivers of Patients With Advanced Cancer: Outcomes From the ENABLE III Randomized Controlled Trial. J Clin Oncol 2015;33:1446-52.

33. Bakitas M, Lyons K, Hegel M, et al. Effects of a Palliative Care Intervention on Clinical Outcomes in Patients With Advanced Cancer. The Project ENABLE II randomised control trial. JAMA 2009;302(7):741.

34. Maltoni M, Scarpi E, Dall'Agata M, et al. Early Palliative Care Italian Study Group (EPCISG). Systematic versus on-demand early palliative care: results from a multicentre, randomised clinical trial. Eur J Cancer 2016;65:61-8.

35. Office for National Statistics, National Survey of Bereaved People (VOICES): England, 2015 [cited 2017 Mar 15]. Available from: https://www.ons.gov.uk/peoplepopulationandcommunity/healthandsocialcare/healthcares ystem/bulletins/nationalsurveyofbereavedpeoplevoices/england2015\#preferences-andchoice-at-the-end-of-life

36. Lutz S, Norrell R, Bertucio C, et al. Symptom Frequency and Severity in Patients with Metastatic or Locally Recurrent Lung Cancer: A Prospective Study Using the Lung Cancer Symptom Scale in a Community Hospital. J Palliat Med 2001;4(2):157-65. 
37. Maione P, Perrone F, Gallo C, et al. Pretreatment Quality of Life and Functional Status Assessment Significantly Predict Survival of Elderly Patients With Advanced Non SmallCell Lung Cancer Receiving Chemotherapy: A Prognostic Analysis of the Multicenter Italian Lung Cancer in the Elderly Study. J Clin Oncol 2005;23(28):6865-72.

38. Basch E, Deal AM, Dueck AC, et al. Survival Results of a Trial Assessing PatientReported Outcomes for Symptom Monitoring During Routine Cancer Treatment. JAMA 2017;318(2):197-8.

39. Basch E, Deal AM, Kris MG, et al. Symptom Monitoring With Patient-Reported Outcomes During Routine Cancer Treatment: A Randomized Controlled Trial. J Clin Oncol 2016;34(6):557-65.

40. Office for National Statistics. National Survey of Bereaved People (VOICES). London: Office for National Statistics, 2015.

41. Public Health England. National End of Life Care Intelligence Network. Number and proportion of deaths by place of occurrence. London: Public Health England, 2015.

42. National Institute for health and Care Excellence. NICE Guidance: Judging whether public health interventions offer value for money. London: NICE, 2013

43. Brumley R, Enguidanos S, Jamison P, et al. Increased Satisfaction with Care and Lower Costs: Results of a Randomized Trial of In-Home Palliative Care. J Am Geriatr Soc 2007;55(7):993-1000.

44. Marie Curie. The hidden challenges of palliative cancer care, 2016. London: Marie Curie, 2016

45. Gomes B, Calanzani N, Curiale V, McCrone P, Higginson I. Effectiveness and costeffectiveness of home palliative care services for adults with advanced illness and their caregivers. Cochrane Database Syst Review 2013 Jun 6;(6):CD007760. 
46. Greer J, Tramontano A, McMahon P, et al. Cost Analysis of a Randomized Trial of Early Palliative Care in Patients with Metastatic Non Small-Cell Lung Cancer. J Palliat Med 2016;19(8):842-8.

47. Normand C. Measuring Outcomes in Palliative Care: Limitations of QALYs and the Road to PalYs. J Pain Symptom Manage 2009;38(1):27-31.

48. UK Clinical Research Collaboration. UK Health Research Analysis 2014. London: Medical Research Council, 2014.

49. Hui D, De La Cruz M, Mori M, et al. Concepts and definitions for 'supportive care,' 'best supportive care,' 'palliative care,' and 'hospice care' in the published literature, dictionaries, and textbooks. Support Care Cancer 2012;21(3):659-85.

50. EPEC-O, education in palliative and end-of-life care for oncology [electronic resource]. U.S. Department of Health and Human Services, National Institutes of Health, National Cancer Institute, American Society of Clinical Oncology, Hospice and Palliative Nurses Association. U.S. Department of Health and Human Services, National Institutes of Health, National Cancer Institute, 2006. 


\section{Figures}

Figure 1: A timeline demonstrating the differences in care plotted against disease progression resulting in death. Adapted from Hui, D et al. ${ }^{49}$

Figure 2: Referral criteria to access SPC. Adapted from APM Commissioning guidance for specialist care. ${ }^{24}$

Figure 3: Mood assessment scores in standard care compared to early palliative care. Adapted from Temel et al. ${ }^{20}$

Figure 4: Mean cost of care in standard care compared to early palliative care. Adapted from Brumley et al. $^{43}$

Figure 5: Proposed involvement of healthcare disciplines, plotted against disease progression. Adapted from the National Cancer Institute. ${ }^{50}$ 


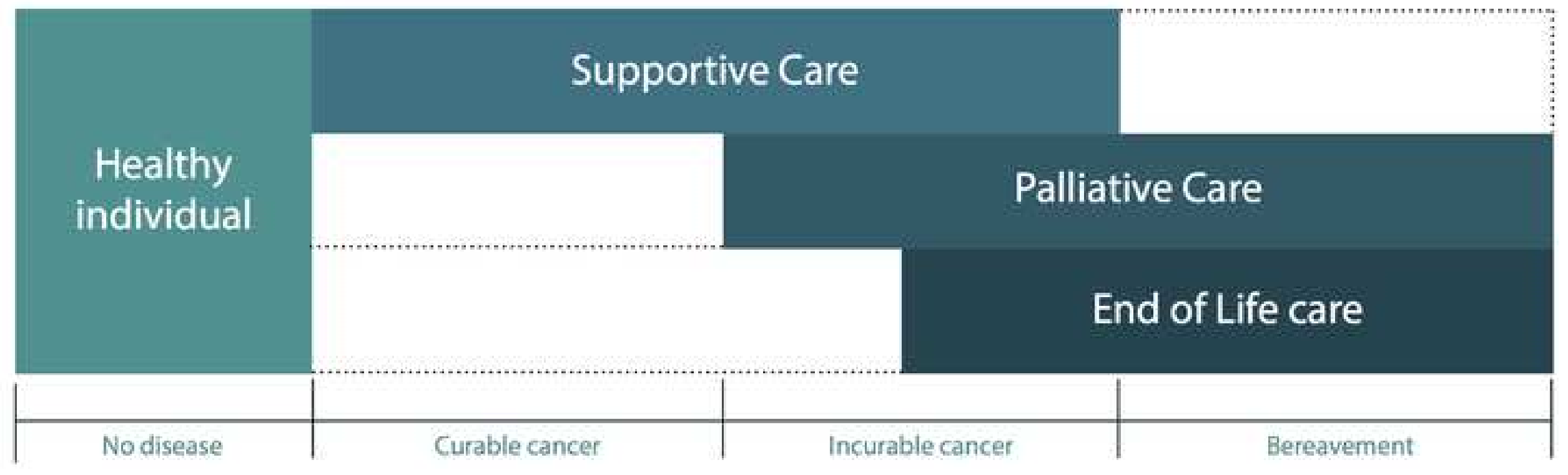




\section{(1)}

Patient has active, progressive advanced disease, a limited prognosis.

Focus of care is on quality of life.

\section{(2)}

Patient has unresolved, complex needs that cannot be met by caring

team.

Could be physical/psychological/ social/spiritual.

\section{(3)}

Patient has given consent for referral. Providing they have capacity. 


\section{$50 \%$}

Standard Care

Early Palliative Care

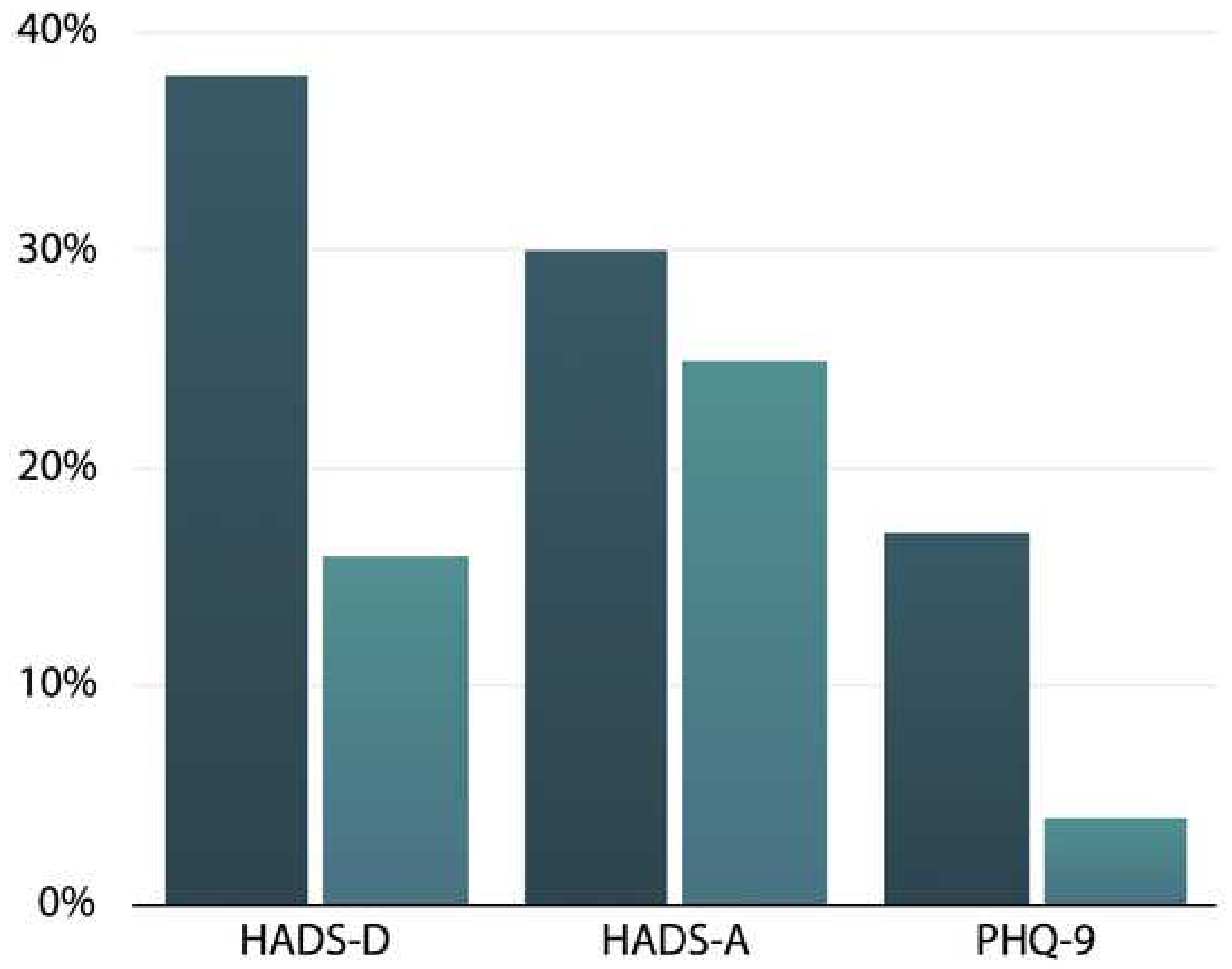




\section{US\$250.00}

US\$200.00

US\$150.00

US\$100.00

US\$50.00

US\$0.00

- Standard Care

- Early Palliative Care 


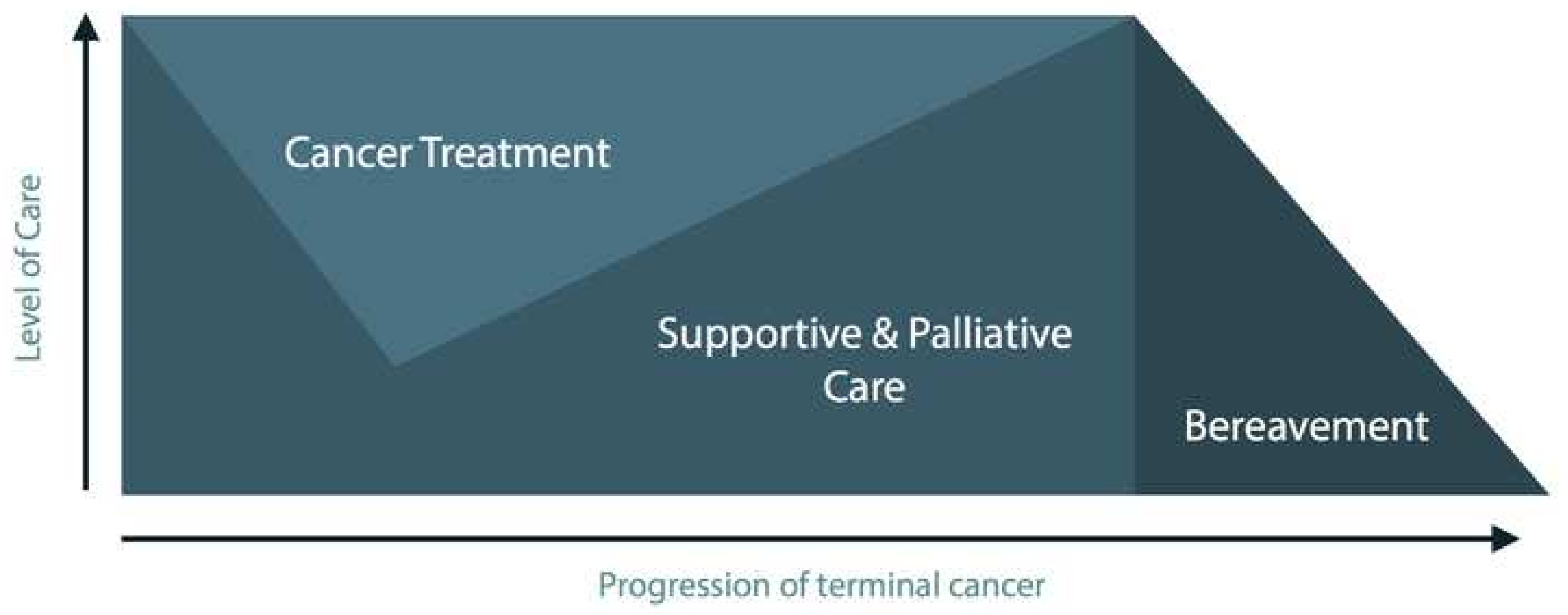




\title{
Response to reviewers' comments
}

Editor: Colleagues, I agree that Appendices and Table 1 should be removed. I look forward to receiving your revised manuscript. The appendices and table 1 have been removed.

Reviewer \#2: Thank you for submitting this manuscript for review. It is well-written and, I believe, has the potential to make a valuable contribution to the literature. The following feedback is offered to strengthen that contribution, as there are some minor revisions required. Thank you for your kind comments!

General Comments

I wonder if text in this manuscript has perhaps been adapted from a student project report or thesis/dissertation, as there are some minor things that require a different approach when publishing a journal article. Eg. the appendices referred to throughout do not appear appropriate or necessary for a journal article of this type. Suggest removing Appendix 1 and Appendix 2. Appendix 2 is not referred to in-text, but please also remove reference to Appendix 1 (in parentheses on p.6). The appendices, and reference to them, have been removed.

Similarly, I think it best to avoid reproducing other authors' quantitative data in the form of tables for a review paper such as this. The point being made here (from a narrative literature review standpoint) would be better made in prose. Suggest removing Table 1 (and reference to this in text on p.7). Table 1 has been removed.

Please also note that each of the Figures (content or relevance) should be introduced or otherwise better incorporated into the text for the reader. See for example '(Figure 1)' on page 4. This approach has been added.

\begin{abstract}
From the title and Abstract there is no indication for the reader as to what type of paper this is. If this is a review paper, I suggest framing the paper to indicate this in the abstract making clear that a review of the literature was conducted. For example, the final para could begin with 'In reviewing the literature, it is clear that...' or words to that effect. This has been added.
\end{abstract}

\section{MAIN TEXT}

Introduction - for me, the introduction could better introduce the reader to the content to follow - by way of a brief outline or the like, to sign-post for the reader any relevant background and what is to come in the paper. This would better prepare the reader for headings such as 'Drivers', 'SPC Guidelines', 'QoL Assessments' 'Symptom Management', 'Effects on Survival' and 'Financial Considerations' etc., rather than having to navigate their context as they read. An additional paragraph has been added to include this.

Please consider the multidisciplinary readership of the journal and cater to this in your writing. For example, specialist palliative care as a 'medical specialty' (p.3) does not appear inclusive of the other healthcare professional disciplines that form an important part of a specialist palliative care team (nursing/allied health/spiritual or pastoral care etc). I appreciate that palliative medicine is a distinct medical specialty, but medicine represents just one of the 
healthcare disciplines that provide palliative care. I suggest rephrasing to better reflect this. We have tried to use terms such as 'healthcare professionals', etc., throughout.

Please use acronyms only if/where they are used more than once. Eg. SPC AND NICE are fine, but 'NCI' is not used more than once. This has been amended.

'Quality of life (QoL) is the mainstay of the SPC approach' - given that palliative care is defined by WHO as 'an approach', this would read much better as '...the mainstay of SPC (please remove 'approach' here). This has been amended.

\section{Conclusion}

As with the introduction, I think structurally, this (important) part of the paper could be strengthened. Not by repeating everything again, but by perhaps offering the reader a broad summary-level recap of what has been covered (this would correspond with the outline provided at the end of the introduction), along with the concluding points that are already provided. In the context of a review paper (in contrast to a research report of empirical study findings), I think this is important for the reader. This has been amended.

With these minor points addressed, I think this paper will make a valuable contribution to the literature. Best wishes for progressing this work. Many thanks for the very useful feedback and suggestions. 